To list an event in the Calendar, contact Julia Phillips, AT\&T Bell Laboratories, 600 Mountain Avenue IE-431, Murray Hill, NJ 07974; telephone (201) 582-4428.

$\mathrm{M}[\mathrm{R}] \mathrm{S}$ indicates the meeting is sponsored by or affiliated with the Materials Research Society.

See MRS BULLETIN Vol. XIII No. 1 for Calendar events from February 15, through March 31 , 1988.

\section{APRIL 1988}

4-8

Technical Symposium on Optics Electro-Optics, and Sensors Orlando, FL

SPIE, P.O. Box 10, Bellingham. WA 98227-0010: (206) 676-3290

5-9

M|R S Materials Research

Society Spring Meeting

Reno, NV

A. Wagner, Materials Research

Society, 9800 McKnight Rd., Suite

327, Pittsburgh, PA 15237;

(412) $367-3003$

10-12

Superconductors in Magnetics-

Commercialization Workshop

San Francisco, CA

Advantage Quest, 110 Sunnyvale-

Saratoga Rd., Suite C2,

Sunnyvale, CA 94087-2515;

(408) 733-0818

13-15

International Conference on

Computer Aided Design in

Composite Materials Technology

Southampton, UK

L. Newman, Computational

Mechanics Institute, 52 Henstead

Rd. Southampton, S01 2DD, UK:

44 (0) 703221398

(See related article in Vol. XII No. 5. )

17-22

Modeling of Casting and Welding

Processes IV

Palm Coast, FL

Engineering Foundation, 345 East

47th St., New York, NY 10017

(212) 705-7835

(See related article in Vol. XII No. 6.)

25-27

Short Course on Rutherford

Backscattering Surface Analysis

Newburyport, MA

C.A. Boyd, General Ionex, $19 \mathrm{Gra}$

Rd., Newburyport, MA 01950;

(617) 462-7147
25-June 17

Spring College in Condensed

Matter on Interaction of Atoms

and Molecules with Solid

Surfaces

Trieste, Italy

ICTP, P.O. Box 586. Strada

Costiera 11,34100 Trieste, Italy

39-40-2240-1

$27-29$

10th Symposium on Applied

Surface Analysis

Lakewood, CO

A.W. Czanderna, Solar Energy

Research Institute, Golden, 80401; (303) 231-1240

\section{MAY 1988}

$1-5$

American Ceramic Society

Annual Meeting

Cincinnati, $\mathrm{OH}$

American Ceramic Society, 75

Brooksedge Plaza Dr., Westerville

$\mathrm{OH} 43081-2821$; (614) 890-4700

$9-11$

\section{M|R|S Processing and \\ Applications of High $T_{C}$ \\ Superconductors: Status and \\ Nucleaire, Laboratoire PHASE, \\ 67037 Strasbourg Cedex. France: \\ 88286543}

Prospects (1988 TMS Northeas

Regional Meeting)

New Brunswick, NJ

B. Kamperman. The Metallurgical

Society, 420 Commonwealth Dr.,

Warrendale, PA 15086:

(412) $776-9050$

(See related article in Vol. XII No. 7. )

\section{2-13}

Controlled Release: Science and

Technology 1988

Melbourne, Australia

R.C. Oppenheim, Victorian

College of Pharmacy Ltd., 381

Royal Parade, Parkville, Victoria

3052, Australia; 61-3-387-7222

$15-20$

Spring Meeting of the

Electrochemical Society

Atlanta, GA

Electrochemical Society, $10 \mathrm{~S}$

Main St., Pennington

NJ 08534-2896

\section{5-21}

NATO Advanced Study Institute

on Heterostructures on $\mathrm{Si}$

Corsica, France

Y.I. Nissim, CNET Bagneux, 196 Avenue H Ravera, 92220 -

Bagneux, France

16-19

Frontiers of Electron Microscopy:

Surfaces and Interfaces

Oak Brook, IL

The Metallurgical Society, 420

Commonwealth Dr. Warrendale.

PA 15086; (412) 776-9050

21-25

American Physical Society

Meeting

New Orleans, LA

W. Havens, 335 E. 45 th St., New

York, NY 10017; (212) 682-7341
30-1

Topical Meeting on Three-

Dimensional Integration

Miyagi, Japan

Y. Hayasaka, Research \&

Development Association for

Future Electron Devices, Fukide

Bldg. No. 2, 4-1-21 Toranomon,

Minato-ku, Tokyo, 105 Japan; 81-

03-434-3871

30-3

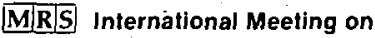

Advanced Materials

Tokyo, Japan

M. Doyama or S. Somiya, Nikkan

Bureau, 8-10, Kudan Kita,

1-chome, Chiyoda-ku, Tokyo 102

Japan; telex: NIKKANKOJ29687

U.S. Coordinator: R.P.H. Chang,

Dept. of Materials Science \&

Engineering, Northwestern

University, Evanston, IL 60208

31-3

\section{E- $\underline{\mathbf{M}|\mathbf{R}| \mathrm{S}}$ E-MRS Meeting}

Strasbourg, France

$P$. Siffert, Centre de Recherches

\section{JUNE 1988}

1-3

Fifth Conference on Semi-

Insulating III-V Materials

Malmo, Sweden

P. Omling, Dept. of Solid State

Physics, University of Lund, Box

118, S-221 00 LUND. Sweden:

2-4

Topical Meeting on High-

Temperature Superconducting

Electron Devices

Miyagi, Japan

K. Mizusawa, Research \&

Development Association for

Future Electron Devices, Fukide

Bldg. No. 2, 4-1-21 Toranomon,

Minato-ku. Tokyo, 105 Japan:

81-03-434-3893

5-8

Physical Electronics

Upton, NY

J.W. Davenport, Physics

Department, Bldg. 510A

Brookhaven National Lab, Upton

NY 11973; (516) 282-3789

5-11

American Chemical Society

Annual Meeting

Toronto, Ontario

American Chemical Society, 1155

16 th St. NW, Washington

DC 20036: (202) 872-4600

6-17 (cont'd August 15-19) Short Course in X-Ray Spectrometry Albany, NY

H. Chessin. State University of

New York, Dept. of Physics, 1400

Washington Ave., Albany.

NY 12222; (518) 442-4512
Kogyo Shimbun Ltd. Planning

46-46-107000

48th Annual Conference on
7-10

Seventh International Conference

in Ion Implantation Technology

Kyoto, Japan

T. Takagi, Kyoto University,

Sakyo-ku, Kyoto 606, Japan

12-17

M|R|S Sixth International

Conference on Ion Beam

Modification of Materials

Tokyo, Japan

K. Gamo, Dept. of Electrical

Engineering. Osaka University,

Toyonaka, Osaka 560, Japan

13-15

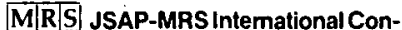

ference on Electronic Materials

Tokyo, Japan

T. Kamiya, Dept. of Electronic

Engineering. University of Tokyo

7-3-1, Hongo, Bunkyo-ku, Tokyo

113, Japan; telex: 2722111 FEUT J

U.S. Coordinator: R.P.H. Chang.

Dept. of Materials Science \&

Engineering, Northwestern

University; Evanston. IL 60208

13-17

Basic Short Course on Scanning

Electron Microscopy and X-ray

Microanalysis

Bethlehem, PA

J.I. Goldstein, Dept of Materials

Science and Engineering. Lehigh

University, Bethlehem, PA 18015

(215) 758-5133

14-16

Second Electronics Materials and

Process Conference

Seattle, WA

SAMPE, 843 West Glentana,

Box 2459, Covina, CA 91722

(818) 331.0616

19-24

11 th International Symposium on

the Reactivity of Solids

Princeton, NJ

M.S. Wittingham, P.O. Box 669

Ridgefield, CT 06877-0669;

(203) 431-5419

(See related article in Vol. XII No. 7.)

20-23

Short Course on Advanced

Topics in Scanning Electron Micros-

copy and X-ray Microanalysis

Bethlehem, PA

J.I. Goldstein, Dept. of Materials

Science and Engineering, Lehigh

University, Bethlehem. PA 18015; (215) 758-5133

20-24

Seventh Penn State Read

Conference on Electrodeposition

University Park, PA

H.W. Pickering, Pennsylvania

State University, 326 Steidle

Bldg., University Park, PA 16802

(814) $863-2640$

20-24

Workshop on Ion Beam

Technology and Equipment 1988

Shanghai, China

S.C. Tsou, Shanghai Institute of

Metallurgy, Shanghai, China 
20-29

Workshop in Advanced Electron

Microscopy

Berkeley, CA

M. Moore, Lawrence Berkeley

Laboratory, Bldg. 72

Berkeley, CA 94720

20-1

Short Course in X-Ray Powder

Diffraction

Albany, NY

$\mathrm{H}$. Chessin State University of

New York, Dept. of Physics, 1400

Washington Ave., Albany,

NY 12222; (518) 442-4512

20-September 9

Research Workshop, in

Condensed Matter, Atomic \&

Molecular Physics

Trieste, Italy

ICTP, P.O. Box 586, Miramare

Strada Costiera 11, 34100 Trieste, Italy; 39-40-2240-1

\section{JULY 1988}

3-15

International School of Materials

Science and Technology 15 th

Course: Solid State Microbatteries

Sicily, Italy

M. Balkanski, Laboratoire de

Physique du Solide, Université

Pierre et Marie Curie, 4 Place

Jussieu, 75230 Paris, Cedex 05

France; 43-36-2525, ext. 4501;

U.S. Coordinator: J.R. Akridge,

Eveready Battery Co., Inc.

Technology Laboratory, P.O. Box

43035, Westlake, OH 44145;

(216) 835-7734

18-21

Fourth International Conference

on the Structure of Non-

Crystalline Materials

Oxnard, CA

C.N.J. Wagner, Dept. of Materials

Science and Engineering, 6531

Boelter Hall, UCLA. Los Angeles.

CA 90024-1595; (213) 825-6265

18-21

Symposium on Carbon Fibers and

Composites

Buffalo, NY

D.D.L. Chung, Dept. of

Mechanical \& Aerospace

Engineering, SUNY, Buffalo,

NY 14260; (716) 636-2520

18-21

16th International Conference on

Quantum Electronics

Tokyo, Japan

IQEC '88 Secretariat, OITDA, 20th

Mori Bldg., 7-4, Nishi-Shimbash

2-chome, Minato-ku, Tokyo 105

Japan; 8135082091

21-22

Short Course on the Fabrication

of Carbon Fiber Polymer-Matrix

Composites

Buffalo, NY

D.D.L. Chung, State University of New York, Dept. of Mechanical \& Aerospace Engineering, Buffalo. NY 14260; (716) 636-2520
$25-29$

International Conference on Magnetism

Paris, France

P. Beauvillain, Comité Local ICM

Exposition, Institut d'Electronique

Fondamentale, Bât. 220 -

Université Paris Sud, 91405 ,

Orsay Cedex, France: 33-1-69-4165-77

31-5

Advances in Cement Manufacture and Use

Henniker, NH

Engineering Foundation, 345 East

47th St., New York, NY 10017;

(212) 705-7835

\section{AUGUST 1988}

$2-4$

International Metals and Metals

Processing Conference-Space

Age Metals and Technology

Dayton, $\mathrm{OH}$

SAMPE, 843 West Glentana,

Box 2459, Covina, CA 91722;

(818) 331-0616

8-12

Fifth Trieste Semiconductor

Symposium and Fourth

International Conference on

Superlattices, Microstructures

and Microdevices

Trieste, Italy

G. Dohler, University of Erlangen,

Erwin-Rommel-Str. 1, 852

Erlangen, W. Germany

49-9131-857294

14-19

32nd Annual International

Technical Symposium on Optical

and Optoelectronic Applied

Science and Engineering

San Diego, CA

SPIE, P.O. Box 10, Bellingham,

WA 98227-0010; (206) 676-3290

15-19

19 th International Conference on

Physics of Semiconductors

Warsaw, Poland

J. Kossut, Institute of Physics,

Polish Academy of Science,

Al. Lotnikow $32 / 46$

02-668 Warsaw, Poland

24-26

International Conference on Solid

State Devices and Materials

Tokyo, Japan

Business Center for Academic

Societies Japan, Yamazaki BIdg.

4F, 40-14, Hongo 2-chome,

Bunkyo-ku, Tokyo 113, Japan;

038175831

28-10

Structure-Property Relationships in Surface Modified Ceramics,

Theory and Applications

NATO Advanced Study Institute

Pisa, Italy

C.J. McHargue, Metals and

Ceramics Division, Oak Ridge

National Laboratory, P.O. Box $X$

Oak Ridge, TN 37831-6118:

(615) $574-4344$

(See related article in Vol. XII No. 5.)
29-1

11 th IEEE Internationa

Semiconductor Lasers

Conference

Boston, MA

R.T. Wangeman, IEEE-LEOS, 345

ast 47th Street, New York

NY 10017; (212) 705-7943

29-2

International Conference on

Defects in Insulating Crystals

Parma, Italy

R. Capelletti, Dept di Fisica, Via

M. D'Azeglio 85, 43100 Parma, Italy

29-2

E-M]R Polycrystalline

Semiconductors '88 - Physical

Properties of Grain Boundaries

and Interfaces

Malente, W. Germany

H.J. Moller and H.P. Strunk

Technische Universitat Hamburg-

Harburg, Eissendorfer Strasse 42,

2100 Hamburg 90, W. Germany

\section{SEPTEMBER 1988}

4-16

NATO Summer School on Plasma

Surface Interactions and

Processing of Materials

Alicante, Spain

O. Auciello, North Carolina State

University, Dept of Nuclear

Engineering. Raleigh, NC 27695

7907: (919) 737-7662

6-9

International Symposium and

Exhibition on Fiber Optics.

Optoelectronics, and Laser

Applications in Science and

Engineering

SPIE, P B Box 10, Bellingham

WA 98227-0010; (206) 676-3290

12-16

International Conference on

Surface Modification of Metals by Ion Beams

Riva Del Garda, Italy

Secretary, IRST, 38050 Povo.

Trento, Italy; 39-0461-810-105

13-15

Third Specialty Polymers

Conference

Cambridge, UK

M. Heald, Butterworth Scientific

Ltd., P.O. Box 63, Westbury

House Bury St., Guildford, Surrey,

GU2 5BH, UK: 44-0483-31261

19-23

International Congress on Optical

Science and Engineering

Hamburg, West Germany

SPIE, P.O. Box 10, Bellingham

WA 98227-0010; (206) 676-3290

26-30

1988 World Materials Congress

Chicago, IL

$V$ Laxmanan, NASA Lewis

Research Center, M.S. 105-1,

Cleveland, $\mathrm{OH} 44135$

(216) 433-3268

26-30

Fourth International Colloquium

on Welding and Melting by

Electron and Laser Beams

Cannes, France

Commissariat a l'Energie

Atomique, DTECHSTA, 91191

Gif-sur-Yvette Cedex, France;

33-1-6908-5825

27

Annual Meeting of the Titanium

Development Association

Chicago, IL

F.W. Rickenbach, 11 W.

Monument Ave., Dayton, $\mathrm{OH}$

45401; (513) 223-8008

$27-29$

20th International Technical

Conference - Materials and

Processes: The Interceptor Point

Minneapolis, MN

M. Smith, SAMPE, 843 West

Glentana, P.O. Box 2459, Covina

CA 91722 ; $(818)$ 331-0616

\section{OCTOBER 1988}

2-7

35 th National Vacuum Symposium Atlanta, GA

N. Hammond, American Vacuum

Society, 335 E. 45th St.

New York, NY 10017

9-14

Electrochemical Society Fall

Meeting

Chicago, IL

Electrochemical Society, $10 \mathrm{~S}$.

Main St., Pennington, NJ

08534-2896

10-13

\section{M|RTS Sclentific Basis for}

Nuclear Waste Management XII

Berlin, West Germany

M. Fuchs, Hahn-Meitner Institut,

Berlin GmbH, Glienicker Strasse

100, D-1000 Berlin 39.

W. Germany; (30) 8009-2291

23-26

Symposium on High Technology

Materials Characterization, in

conjunction with the Pacific Coast

Regional Meeting of the American

Ceramic Society

San Francisco, CA

Wayne S. Young. IBM

Corporation, 5600 Cottle Ro.

San Jose CA 95193

25-29

TMS Fall Meeting

Chicago, IL

B. Kamperman, The Metallurgical Society, 420 Commonwealth Dr.

Warrendale, PA 15086:

(412) $776-9050$

\section{NOVEMBER 1988}

28-3

M|R/S. Materials Research

Society Fall Meeting 